\title{
Dynamic Spectrum Access Protocol Without Power Mask Constraints
}

\author{
Haythem Bany Salameh and Marwan Krunz \\ Department of Electrical and Computer Engineering \\ University of Arizona, Tucson, AZ 85721 \\ Email: \{haythem,krunz\}@ece.arizona.edu
}

\author{
Ossama Younis \\ Applied Research, Telcordia Technologies, Inc. \\ Piscataway, NJ 08854 \\ Email: oyounis@ research.telcordia.com
}

\begin{abstract}
In this work, we investigate a statistical approach for dynamic spectrum access and radio resource management (RRM) in opportunistic cognitive radio (CR) networks. We propose a distributed MAC protocol for such networks that enables unlicensed users to dynamically utilize the available spectrum while limiting the imposed interference on primary (PR) users. Our proposed protocol is novel in three aspects. First, it does not require CR users to coordinate with PR users. Second, it does not assume any predefined CR-to-PR power mask, and thus can exploit the available spectrum more efficiently. Third, it provides the PR users with a statistical guarantee on the fraction of time that their reception may be corrupted by $C R$ users. To avoid corrupting PR user receptions, the protocol computes the maximum power that a CR transmission can use based on current network conditions. We show how to compute this maximum power by deriving models for the PR-to-CR and PR-to-PR interference. Simulation experiments illustrate that our MAC protocol can satisfy the statistical guarantee for PR users under various user deployment models and traffic loads.
\end{abstract}

\section{INTRODUCTION}

The widespread acceptance of the unlicensed wireless communication services and applications has significantly increased the demand for more transmission capacity. Accordingly, the unlicensed portions of the spectrum (e.g., the ISM bands) have become increasingly crowded. At the same time, the FCC has recently reported that licensed bands are vastly underutilized [1], [2]. To overcome spectrum scarcity, cognitive radios (CRs) have been proposed to allow opportunistic spectrum access to the licensed spectrum.

A cognitive radio network (CRN) has unique characteristics that distinguishes it from a traditional wireless communication network. The latter allocates spectrum statically, resulting in spectrum wastage, and has a fixed radio functionality. In contrast, a CRN dynamically utilizes the available spectrum and adapts its operating parameters (e.g., carrier frequency, number of channels, etc.) according to the surrounding environment [3], [4]. In an environment where several licensed primary radio networks (PRNs) operate, a network of CR users that co-exists with PR users needs to exploit the underutilized portion of the spectrum. In this case, the crucial challenge is how to allow CR users to share the licensed spectrum with PR users without degrading the performance of the latter. A key performance measure is the "outage probability" $\left(p_{\text {out }}\right)$ of a PR user, defined as the fraction of time during which the total interference power at a PR receiver exceeds the maximum tolerable interference.

One of the key challenges to enabling CR communications is how to perform opportunistic medium access control while limiting the interference imposed on PR users. Recently, several attempts were made to develop MAC protocols for CRNs (e.g., [5]-[11]). Existing work on spectrum sharing/access protocols can be classified according to their architecture (centralized or decentralized), spectrum allocation behavior (cooperative or non-cooperative), and spectrum access technique (overlay or underlay) [2]. In [9], [10] centralized protocols were proposed for coordinating spectrum access. For an ad hoc CRN without centralized control, it is desirable to have a distributed
MAC protocol that allows every CR user to individually access the spectrum.

The FCC defined the interference temperature model [3], which provides a metric for measuring the interference experienced by PR users. Clancy [12] used this model to select an optimal bandwidth/power assignment for CRs. However, no operational protocol was proposed, as was the case with several other proposals. In addition, some proposed protocols (e.g., [5], [13], [14]) do not provide guarantees on the performance of PR users and require a pre-specified power mask for CR transmissions. We refer the interested reader to our technical report [15] for more details and examples of related work.

Note that a number of multi-channel contention-based MAC protocols were also proposed in the context of CRNs (e.g., [5][8], [14], [16]). AS-MAC [6] is a spectrum-sharing protocol for CRNs that coexist with a GSM network. CR users select channels based on the CRN's control exchanges and the GSM network's broadcast information. Explicit coordination with the PRNs is required. In [16], the concept of a time-spectrum block is introduced to model spectrum reservation. Based on this concept, the authors presented a centralized and a distributed CRN protocol with a common control channel for spectrum allocation. To the best of our knowledge, there is no CRN MAC protocol that provides a soft guarantee on the performance of PR users without assuming a predefined CR-to-PR power mask or requiring coordination between $\mathrm{CR}$ and PR users.

Contributions: The contribution of this paper is threefold. First, we develop stochastic models for the PR-to-PR and the PR-to-CR interference under a Rayleigh fading channel model. In the course of constructing these models, we derive closed-form expressions for the mean and variance of the total interference at a receiving node. Closed-form expressions for the characteristic function (CF) of such interference are also obtained for integer-valued path loss exponents. Numerical and simulation results indicate that the resulting distribution of the total interference is well approximated by a lognormal function. Second, based on the developed interference models, we derive an expression for the maximum allowable powers for a CR transmission. The computed powers provide a statistical guarantee on the PRN performance. These powers are then used in the design of a distributed CSMA/CA-based MAC protocol for CRNs. Our protocol does not require online interaction with PRNs. Through local exchange of control messages, the protocol enables a pair of $\mathrm{CR}$ users to select the minimum number of channels to use according to the surrounding interference and the rate demand of the CR transmitter. CR users can communicate over both unused and partially used licensed channels without needing to coordinate with PRNs. Most importantly, our protocol functions without assuming a predefined CR-to-PR interference power mask.

It should be noted that interference modeling in wireless networks was previously studied under the assumption of an infinite user population, operating within an unbounded field. For example, [13], [17] assumed that nodes are distributed according to a Poisson distribution, and characterized the 
distribution of the interference for an idealized infinite-size network operating within an infinite field. No multipath fading was considered. It is easy to show that their model leads to total interference whose mean and variance are infinite. Such a model cannot be applied in our work, as we consider a finite number of users.

The rest of the paper is organized as follows. Section II introduces our system model. In Section III, we develop stochastic models for the PR-to-PR and PR-to-CR interference. Section IV shows how to provide a statistical guarantee on the performance of PR users. We introduce our MAC protocol in Section V and evaluate its performance in Section VI. Finally, Section VII gives concluding remarks.

\section{SySTEM MOdEL}

We consider a hybrid network, consisting of $M$ different PRNs and one (secondary) CRN. The $M+1$ networks co-exist within the same geographical space. The PRNs are licensed to operate on non-overlapping frequency bands. In reality, a PRN may occupy multiple, non-contiguous frequency bands. Such a PRN can be easily captured in our setup by using multiple virtual PRNs, each operating over its own band. For the $i$ th PRN, $i=1,2, \ldots M$, we denote its carrier frequency, channel bandwidth, and maximum transmission power by $f_{i}, B_{i}$, and $P_{t}{ }^{(i)}$, respectively. To make our analysis tractable, we model the locations of users in the $i$ th PRN as a homogeneous Poisson random variable on a disk area of parameter (density) $\rho_{i}$. This model was previously used in [12], [17]. In our simulations, we relax this assumption and consider arbitrary deployment scenarios. Each user in the $i$ th PRN acts as an ON/OFF source. We define the "activity factor" $\alpha_{i}$ as the fraction of time that a user in the $i$ th PRN is ON [18], [19]. Estimating the activity behavior of PR users was investigated in [18], [19]. Specifically, in [18], $\alpha_{i}$ was estimated by maintaining a run length of the idle/busy period for each channel. Whenever the idle duration is ended by a PR transmission, the run length is recorded in a circular buffer. For our purposes, we assume that a similar mechanism for estimating $\alpha_{i}$ is in place. In section VI, we evaluate the impact of inaccurately estimating $\alpha_{i}$.

CR users can opportunistically access the entire spectrum. Each CR user is equipped with $n_{r}$ radio transceivers, $1 \leq$ $n_{r} \leq M$, that can be used simultaneously. The CR user has a wideband sensing capability with a narrowband resolution. Such capability can be achieved using a wideband antenna, a power amplifier, and adaptive filters [2]. Thus, a CR user can sense the available spectrum in one shot (simultaneously sensing several GHz-wide bands [20]) and estimate the instantaneous interference over each band. Such advanced spectrum sensing technology is readily available through a DSP technique called cyclostationary feature detection [2], [20]. Alternatively, a sequential partial sensing approach can be employed at the cost of negligible switching/sensing overhead [8]. It is worth mentioning that off-the-shelf wireless cards (e.g., ICS-572 products [21]) can readily serve as a fully functional wideband multi-channel CR interface that enables a CR user to perform analysis of the RF spectrum (i.e., sensing) in real time.

\section{INTERFERENCE ANALYSIS}

In this section, we develop stochastic models for the PR-to$\mathrm{PR}$ and the PR-to-CR interference. These models are later used to determine the transmission power for CR nodes over each opportunistically seized channel. Without loss of generality, we consider one of the PRNs (PRN $i$ ) and determine the total interference caused by its users at an arbitrary receiver $v$ (primary or cognitive). Because of the highly nonlinear attenuation behavior of typical RF channels, we assume that the interference contributed to $v$ by PR users that lie outside a disk of radius $r_{c}\left(r_{c} \gg d_{o}^{(i)}\right)$ is negligible. This is inline with [22], in which $r_{c}$ was used to indicate the distance of the "first-tier interferers". Our simulations (Section VI) relax this assumption.

\section{A. Wireless Channel Model}

Let $d_{o}{ }^{(i)}$ be the close-in distance for the $i$ th PRN, defined as the distance from a transmitter after which the RF channel can be approximated by the free-space model [23]. We consider a Rayleigh fading model to describe the channel between any two users. Specifically, at a transmission distance $d$, the received power over the $i$ th channel $^{1}$ is given by:

$$
P_{r}^{(i)}=P_{o}^{(i)}\left(\frac{d}{d_{o}{ }^{(i)}}\right)^{-n} \xi^{(i)}, \quad d \geq d_{o}{ }^{(i)}
$$

where $P_{o}^{(i)}=\frac{P_{t}{ }^{(i)} G_{t}^{(i)} G_{r}^{(i)} l_{i}^{2}}{\left(4 \pi d_{o}^{(i)}\right)^{2}}$ is the path loss of the close-in distance $d_{o}^{(i)}, G_{t}^{(i)}$ is the antenna gain of the transmitter, $G_{r}^{(i)}$ is the antenna gain of the receiver, $l_{i}$ is the wavelength of $f_{i}, n$ is the path loss exponent, and $\xi^{(i)}$ is a normalized random variable that represents the power gain of the fading process. For a Rayleigh fading, $\xi^{(i)}$ is exponentially distributed; $\operatorname{Pr}\left\{\xi^{(i)} \leq\right.$ $y\}=1-e^{-y}[23]$.

According to [23], $d_{o}{ }^{(i)}$ is given by $d_{o}{ }^{(i)}=$ $\max \left\{\frac{2 D^{2}}{l_{i}}, D, l_{i}\right\}$, where $D$ is the antenna length. In practice, $d_{o}{ }^{(i)}$ is of the same order of magnitude as the node's dimensions. For an 802.11 card operating in the 2.4 $\mathrm{GHz}$ band (5cm-long antenna), $d_{o}{ }^{(i)}=12 \mathrm{~cm}$. Accordingly, it is reasonable to assume that $\operatorname{Pr}\left\{d<d_{o}{ }^{(i)}\right\}$ is very small.

\section{B. PR-to-CR Interference}

We now derive the statistics of the aggregate interference from the $i$ th $\mathrm{PRN}$ on a given $\mathrm{CR}$ receiver. Denote such interference by $P_{P R-C R}^{(i)}$. Approximately, this is equal to the sum of the interference powers of all active PR transmitters within radius $r_{c}$ of the $\mathrm{CR}$ receiver, i.e., $P_{P R-C R}^{(i)} \approx \sum_{j} P_{r, j}^{(i)}$, where $P_{r, j}^{(i)}$ is the received power associated with the $j$ th active PR transmitter of the $i$ th channel, and the summation is carried out over all active PR transmitters in a disc of radius $r_{c}$.

Before proceeding further, we need to determine the distribution of the distance between a PR transmitter and a CR/PR receiver. Let $K_{i}$ denote the (random) number of potential PR interferers within the interfering area $R_{c}$, where $R_{c}=\pi r_{c}^{2}$. We assume that PR users are randomly located according to a Poisson distribution. Then,

$$
\operatorname{Pr}\left\{K_{i}=k_{i}\right\}=\frac{e^{-\rho_{i} R_{c}}\left(\rho_{i} R_{c}\right)^{k_{i}}}{k_{i} !}, \quad k_{i}=0,1,2, \ldots
$$

The distribution of the locations of the $k_{i}$ interferers is that of $k_{i}$ independent and identically distributed (i.i.d.) uniform random variables $[17]^{2}$. Thus, the pdf of the distance $r$ between a receiver at the center of $R_{c}$ and an interferer that is randomly located inside $R_{c}$ is given by [17]:

$$
f_{R}(r)= \begin{cases}\frac{2 r}{r_{c}^{2}}, & r \leq r_{c} \\ 0, & \text { otherwise }\end{cases}
$$

\footnotetext{
${ }^{1}$ Because of our assumed 1-to-1 mapping between the PRNs and the channels, the index $i$ is used to refer to either one.

${ }^{2}$ In our simulations (Section VI), we study the performance of our protocol under both uniform and skewed user distributions.
} 
We assume that different interfering transmissions experience i.i.d. fades. This assumption is justified by noting that the distance between any two PR interferers is typically much larger than the wavelength of the carrier frequency of a PRN (e.g., for a PRN operating at $900 \mathrm{MHz}, l_{i}=33 \mathrm{~cm}$ ).

Theorem 1: The characteristic function (CF) for the aggregate PR-to-PR interference power from the $i$ th $\mathrm{PRN}$ is approximately given by:

$$
\begin{aligned}
& \phi_{P_{P R-C R}^{(i)}}(\omega) \approx \\
& \exp \left(\alpha_{i} \rho_{i} R_{c}\left[\left(2\left(\frac{d_{o}^{(i)}}{r_{c}}\right)^{2} I_{C R, i}(n, \omega)\right)-1\right]\right) .
\end{aligned}
$$

where $I_{C R, i}(n, \omega) \stackrel{\text { def }}{=} \int_{1}^{\frac{r_{c}}{d_{o}^{(i)}}} \frac{x^{n+1}}{x^{n}-j w P_{o}^{(i)}} d x$.

Proof: See Appendix.

For integer values of $n, I_{C R, i}(n, \omega)$ has a closed-form solution [24], and thus, the CF in (4) has a closed-form expression. For the case of a non-integer $n, I_{C R, i}(n, \omega)$ can be evaluated numerically.

Using the fact that $\frac{d_{o}^{(i)}}{r_{c}} \ll 1$, we arrive at the following approximate expressions for the mean and variance of $P_{P R-C R}^{(i)}$ :

$$
\begin{aligned}
& \mathrm{E}\left[P_{P R-C R}^{(i)}\right] \stackrel{\text { def }}{=} \phi_{P_{P R-C R}^{(i)}}^{\prime}(0) \approx \\
& \begin{cases}\frac{2 \pi \alpha_{i} \rho_{i} P_{o}^{(i)} d_{o}^{(i)^{2}}}{(2-n)} e^{-\pi \alpha_{i} \rho_{i} d_{o}^{(i)^{2}}}\left[\left(\frac{r_{c}}{d_{o}^{(i)}}\right)^{2-n}-1\right], & 1 \leq n<2 \\
2 \pi \alpha_{i} \rho_{i} P_{o}^{(i)} d_{o}^{(i)^{2}} e^{-\pi \alpha_{i} \rho_{i} d_{o}^{(i)^{2}}} \ln \left[\frac{r_{c}}{d_{o}^{(i)}}\right], & n=2 \\
\frac{2 \pi \alpha_{i} \rho_{i} P_{o}^{(i)} d_{o}^{(i) 2}}{n-2} e^{-\pi \alpha_{i} \rho_{i} d_{o}^{(i)^{2}}}, & n>2\end{cases} \\
& \text { and } \operatorname{var}\left(P_{P R-C R}^{(i)}\right) \approx \\
& \begin{cases}\frac{\pi \alpha_{i} \rho_{i}}{(n-1)}\left[2 P_{o}^{(i)} d_{o}^{(i)} e^{-\pi \alpha_{i} \rho_{i} d_{o}^{(i) 2}}\right]^{2}, & n>1 \\
2 \pi \alpha_{i} \rho_{i}\left[2 P_{o}^{(i)} d_{o}^{(i)} e^{\left.-\pi \alpha_{i} \rho_{i} d_{o}^{(i)}\right]^{2}} \ln \left(\frac{r_{c}}{d_{o}^{(i)}}\right),\right. & n=1 .\end{cases}
\end{aligned}
$$

Note that the above approximations for the mean and the variance show no dependence on $r_{c}$ for $n>2$.

While a closed-form expression for the pdf of $P_{P R-C R}^{(i)}$ cannot be found, numerical inversion of the $\mathrm{CF}$ and empirical fitting of the simulated data (Section III-D) show that this pdf is well approximated by the lognormal distribution.

\section{PR-to-PR Interference}

In addition to estimating the PR-to-CR interference, our design requires a $\mathrm{CR}$ user to estimate the PR-to-PR interference power, denoted by $P_{P R-P R}^{(i)}$ for the $i$ th PRN, so that an upper bound on the $\mathrm{CR}$ transmission power can be computed while providing a guarantee on the outage probability for PR users. Let $b_{i}$ denote the minimum distance between a PR receiver and the nearest PR interferer ${ }^{3}$. This value is technology-dependent and is fixed for a given PRN. For example, in a cellular network in which adjacent cells do not use common frequencies, $b_{i}$ is the minimum reuse distance, defined as the minimum distance between a base station of a cell and a mobile terminal of another non-adjacent cell that guarantees acceptable link quality. This value can be easily shown to be equal to the diameter of a cell.

To characterize the PR-to-PR interference, we use a similar methodology to that used in the previous section. We replace

\footnotetext{
${ }^{3}$ Mitigating interference over band $i$, and consequently achieving successful communications, necessitates imposing a minimum distance between active PR users.
}

the lower integration limit in (20) by $b_{i}$. The CF of PR-to-PR interference is thus given by:

$$
\begin{aligned}
& \phi_{P_{P R-P R}^{(i)}}(\omega) \stackrel{\text { def }}{=} E\left[e^{j \omega P_{P R-P R}^{(i)}}\right] \approx \\
& \exp \left(\alpha_{i} \rho_{i} R_{c}\left[\left(2\left(\frac{d_{o}^{(i)}}{r_{c}}\right)^{2} I_{P R, i}(n, \omega)\right)-1\right]\right)
\end{aligned}
$$

where $I_{P R, i}(n, \omega) \stackrel{\text { def }}{=} \int_{\frac{b_{i}}{d_{o}^{(i)}}}^{\frac{r_{c}}{d^{(i)}}} \frac{x^{n+1}}{x^{n}-j \omega P_{o}^{(i)}} d x$.

Consequently, the mean and variance for the PR-to-PR interference are approximately given by:

$$
\begin{aligned}
& \mathrm{E}\left[P_{P R-P R}^{(i)}\right] \approx \\
& \int \frac{2 \pi \alpha_{i} \rho_{i} P_{o}^{(i)} d_{o}^{(i) 2} e^{-\pi \alpha_{i} \rho_{i} b_{i}^{2}}}{(2-n)\left(d_{o}^{(i)}\right)^{2-n}}\left[r_{c}{ }^{2-n}-b_{i}{ }^{2-n}\right], \quad 1 \leq n<2 \\
& 2 \pi \alpha_{i} \rho_{i} P_{o}^{(i)} d_{o}^{(i)} e^{2 \pi \alpha_{i} \rho_{i} b_{i}^{2}} \ln \left[\frac{r_{c}}{b_{i}}\right], \quad n=2 \\
& \frac{2 \pi \alpha_{i} \rho_{i} P_{o}^{(i)} d_{o}^{(i)}{ }^{2}}{n-2} e^{-\pi \alpha_{i} \rho_{i} b_{i}^{2}}\left(\frac{b_{i}}{d_{o}^{(i)}}\right)^{2-n}, \quad n>2 \\
& \text { and } \operatorname{var}\left(P_{P R-P R}^{(i)}\right) \approx \\
& \begin{cases}\frac{\pi \alpha_{i} \rho_{i}}{(n-1)}\left[2 P_{o}^{(i)} d_{o}^{(i)} e^{-\pi \alpha_{i} \rho_{i} b_{i}^{2}}\right]^{2}\left(\frac{b_{i}}{d_{o}^{(i)}}\right)^{2(1-n)}, & n>1 \\
2 \pi \alpha_{i} \rho_{i}\left[2 P_{o}^{(i)} d_{o}^{(i)} e^{-\pi \alpha_{i} \rho_{i} b_{i}^{2}}\right]^{2} \ln \left(\frac{r_{c}}{b_{i}}\right), & n=1 .\end{cases}
\end{aligned}
$$

Similar to the case of PR-to-CR interference, for integer values of $n$, the $\mathrm{CF}$ in (7) has a closed-form expression. Furthermore, we found that the lognormal function well approximates the distribution of $P_{P R-P R}^{(i)}$.

\section{Model Verification}

We use simulations to empirically verify the validity of the derived PR-to-CR interference model (results for the validation of the PR-to-PR interference model are similar). We consider 6) a circular field of radius 100 meters in which the users of four PRNs are uniformly distributed. The transmission power for a PR user is 1 Watt. The antenna length $(D)$ is $5 \mathrm{~cm}$. Time is divided into slots. At any given slot, each user in PRN $i$ transmits with probability $\alpha_{i}$. Experiments are performed for different numbers of PR users. Channels used are in the 900 $\mathrm{MHz}, 1.5 \mathrm{GHz}, 2.4 \mathrm{GHz}$, and $4.0 \mathrm{GHz}$ bands. The activity factors are 0.6, 0.5, 0.4, and 0.2. First, we assess the goodness

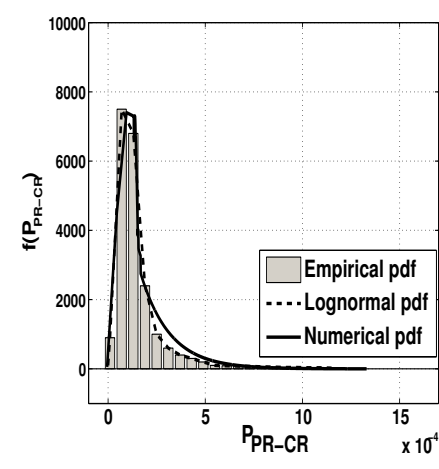

(a) Histogram.

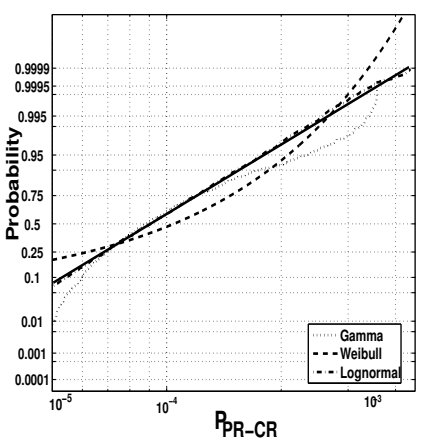

(b) Data-probability plot.
Fig. 1. Interference model verification when $n=2$.

of the approximations in (5) and (6) for the mean and variance of $P_{P R-C R}^{(i)}$. The results (shown in our technical report [15]) indicate that the derived expressions well approximate the measured statistics, with less than $1 \%$ error. 
To validate the conjecture that the distribution of the interference model is well approximated by a lognormal distribution, we compute the pdf of the interference for PRN 1 with $n=2$ in two ways: (1) by constructing the histogram of the simulated data, and (2) by numerically inverting the CF in (4). Figure 1(a) plots the empirical and numerically computed pdfs of $P_{P R-C R}^{(1)}$ against the theoretical lognormal distribution with mean and variance given in (5) and (6), respectively. Visual inspection of the figure indicates the adequacy of the lognormal distribution. Figure 1(b) shows the probability plot of the empirical data against three different distributions: Gamma, Weibull, and Lognormal. In this figure, only the plotted points that correspond to the lognormal function reasonably give straight lines and follow the empirical distribution fairly closely. Similar behaviors were observed for other PRNs and different values of $n$ (see [15] for details).

\section{Guaranteeing Outage Probability for PR USers}

Typical PRNs (e.g., cellular networks, TV broadcast networks) support voice- and video-based services. Such services can tolerate "short" infrequent interruptions (i.e., outages) without significant impact on the perceptual audio/video quality ${ }^{4}$. Motivated by this fact, we advocate supporting soft outage guarantees for PR users. More specifically, we determine the maximum transmission powers for $\mathrm{CR}$ users over various channels such that the outage probability $\left(p_{\text {out }}\right)$ at a PR receiver is guaranteed to be below a given constant $\beta$, where $0<\beta \ll$ 1. Let $P_{C, \beta}^{(i)}$ be the maximum allowable transmission power that a CR transmitter can use over channel $i$ such that all communicating PR users within the communication range of the transmitting $\mathrm{CR}$ are not impacted by this transmission with probability $1-\beta$. We enforce an exclusive channel occupancy policy on CR transmissions, whereby a channel occupied by a $\mathrm{CR}$ user cannot be simultaneously allocated to another CR user in the same vicinity (inline with the CSMA/CA mechanism). Accordingly, we compute an upper bound on the amount of interference that can be introduced by a CR transmitter over each channel. Consider the $j$ th PR user of the $i$ th PRN. With probability $1-\beta$, the following condition should be satisfied by every CR user:

$$
\begin{array}{r}
P_{P R-P R, j}^{(i)}+g_{C, j}^{(i)} P_{C, \beta}^{(i)} \leq P_{L}^{(i)}, \\
\forall j=1,2, \ldots, \text { and } \forall i=1,2, \ldots, M
\end{array}
$$

where $P_{P R-P R, j}^{(i)}$ is the total PR-to-PR interference power measured at the $j$ th PR receiver of the $i$ th PRN, $g_{C, j}^{(i)}$ is the gain between the given CR transmitter and the $j$ th PR receiver, and $P_{L}^{(i)}$ is the interference power limit of a PR receiver in the $i$ th PRN. The value of $P_{L}^{(i)}$, which is sometimes referred to as the load or interference margin, interference temperature, etc., is typically known for a given PRN (e.g., set by the FCC) [3], [12].

Because we assume no active coordination between $\mathrm{CR}$ and PR users, $g_{C, j}^{(i)}$ is difficult to measure at a CR user. An estimate of $g_{C, j}^{(i)}$ that preserves the required bound on $p_{\text {out }}$ is needed to compute $P_{C, \beta}^{(i)}$. In [12], the selection of $g_{C, j}^{(i)}$ was computed based on the shortest distance between a PR receiver and a CR transmitter.

We now derive the CDF of the distance between a CR transmitter and the closest active PR receiver. Based on such a

\footnotetext{
${ }^{4}$ For example, opportunistic WRAN users are only required to support a $90 \%$ detection rate of PR signals [25]
}

$\mathrm{CDF}$ and following the same methodology in [12], we propose a mathematical formulation for selecting a value of $g_{C, j}^{(i)}$ that preserves the target $p_{\text {out }}$. For a given PRN $i$, let the distance between a CR transmitter located at the center of a disk of radius $r_{c}\left(r_{c} \gg d_{o}^{(i)}\right)$ and the closest active PR receiver be denoted by $R_{\text {min }}$. Then, $R_{\text {min }}=\min \left\{R_{j}: j \in \Gamma\right\}$, where $R_{j}$ is a random variable representing the distance between a CR transmitter and the $j$ th PR receiver, and $\Gamma$ is the set of active PR receivers in the given PRN. According to (3), the CDF of $R_{j}$ is given by $F_{R_{j}}(r)=\frac{r^{2}}{r_{c}^{2}}$. Conditioning on active PR receivers $n_{i}$, the CDF of $R_{\min }$ is given by:

$$
1-\prod_{j=1}^{n_{i}}\left[1-F_{R_{j}}(r)\right]=1-\left[1-\left(\frac{r}{r_{c}}\right)^{2}\right]^{n_{i}}
$$

Given that the number of active receivers is a Poisson random variable with parameter $\alpha_{i} \rho_{i}$, the CDF of $R_{\text {min }}$ can be obtained by removing the conditioning in (11) and algebraically manipulating the result to arrive at:

$$
F_{R_{\text {min }}}(r)=1-e^{-\alpha_{i} \rho_{i} \pi r^{2}} .
$$

Let $r^{*}$ denote the distance used in setting $g_{C, j}^{(i)}$ in (10), which is selected based on a target percentage of $F_{R_{m i n}}$ (i.e., $\left.F_{R_{\text {min }}}\left(r^{*}\right)=1-p^{*}\right)$. Formally, with probability $p^{*}$, where $p^{*}$ is very close to one, the distance between a CR transmitter and its closest $\mathrm{PR}$ receiver is at least $r^{*}$. By substituting $F_{R_{m i n}}\left(r^{*}\right)=1-p^{*}$ in (12) and solving for $r^{*}$, we obtain: $r^{*}=\sqrt{\frac{-\ln \left(p^{*}\right)}{\alpha_{i} \rho_{i} \pi}}$.

Depending on the relative location of a PR receiver with respect to a CR transmitter, there are two possible scenarios for outage at a $\mathrm{PR}$ receiver:

- The PR receiver falls within a distance less than $r^{*}$ from a CR transmitter. The likelihood of this scenario is $1-p^{*}$. In this case, we conservatively set $\operatorname{Pr}\left[\right.$ outage $\left.\mid r<r^{*}\right] \approx 1$.

- The PR receiver is at a distance greater than $r^{*}$ from a CR transmitter. The likelihood of this scenario is $p^{*}$. In this case, let $\gamma \stackrel{\text { def }}{=} \operatorname{Pr}\left[\right.$ outage $\left.\mid r>r^{*}\right]$.

Accounting for the above two scenarios, the overall outage probability can be computed via a straightforward application of Bayes's rule, i.e.,

$$
\begin{aligned}
p_{\text {out }}=\operatorname{Pr}\left[\text { outage } \mid r<r^{*}\right] \operatorname{Pr}\left[r<r^{*}\right]+ \\
\operatorname{Pr}\left[\text { outage } \mid r>r^{*}\right] \operatorname{Pr}\left[r>r^{*}\right] \\
=1 \times\left(1-p^{*}\right)+\gamma \times p^{*}=1-(1-\gamma) p^{*} .
\end{aligned}
$$

Recall that we require $p_{\text {out }} \leq \beta$, which implies:

$$
\gamma \leq 1-\left(\frac{1-\beta}{p^{*}}\right)
$$

Note that $\gamma$ cannot be negative. Thus, for a valid bound on $\gamma$, the following constraint must be satisfied: $\left(\frac{1-\beta}{p^{*}}\right)<1$.

Equations (13) and (14) reveal that in order to preserve the required bound on $p_{\text {out }}$ (i.e., $\beta$ ), the condition in (10) should be satisfied by every CR user that is located at a distance greater than $r^{*}$, with probability $1-\gamma$. To satisfy this condition with probability $1-\gamma$, we compute the $(1-\gamma)$ quantile of $P_{P R-P R, j}^{(i)}$, denoted by $P_{\gamma}^{(i)}$. Because $P_{P R-P R}^{(i)}$ is approximately lognormally distributed, its $(1-\gamma)$-quantile is given by:

$$
P_{\gamma}^{(i)}=\exp \left(\sqrt{\operatorname{var}\left(P_{P R-P R}^{(i)}\right)} \Phi^{-1}(1-\gamma)\right)
$$


where $\Phi^{-1}$ is the $(1-\gamma)$-quantile of the standard normal distribution.

By substituting $P_{\gamma}^{(i)}$ in (10) and rearranging the equation, we obtain an upper bound on the interference that a CR transmitter is allowed to contribute to the $i$ th PRN while ensuring $p_{\text {out }} \leq$ $\beta$ :

$$
P_{C, \beta}^{(i)} \leq \frac{P_{L}^{(i)}-P_{\gamma}^{(i)}}{g_{C, j}^{(i)}}, \quad i=1,2, \ldots, M .
$$

Accordingly, the maximum allowable transmission powers for a CR user over various channels are given by the vector $\mathbf{P}_{C, \beta}=$ $\left[P_{C, \beta}^{(1)}, P_{C, \beta}^{(2)}, \ldots, P_{C, \beta}^{(M)}\right]$, computed from the RHS of (16).

\section{THE MAC PRotocol}

Our proposed MAC protocol is distributed and asynchronous. It uses the previous analysis to enable opportunistic CR communications while providing soft guarantees on the performance of PR users. Before we describe the protocol's operation in detail, we first state our main assumptions and define the different transmission regions around a $\mathrm{CR}$ user $A$. These regions describe $A$ 's "view" of its neighborhood.

\section{A. Assumptions}

For each frequency channel, we assume that its gain is stationary for the duration of one data packet. This assumption holds for typical mobility patterns and transmission rates. We assume symmetric channel gains between two CR users. This is a typical assumption in many RTS/CTS-based protocols, including the IEEE 802.11 scheme. Our protocol assumes the availability of a prespecified control channel of Fourier bandwidth $B_{c}$, where $B_{c} \ll B_{i}, i=1, \ldots, M$ (a similar assumption is made in [6], [11], [26]). Such a channel is not necessarily dedicated to the CRN. It may, for example, be one of the unlicensed ISM bands. We assume that out of the $n_{r}$ transceivers, one is dedicated to the control channel, while the other $n_{r}-1$ transceivers can be tuned to any data channels. Our approach follows similar interframe spacings and collision avoidance strategies of the 802.11 protocol (over the control channel) by using physical carrier sense and backoff before initiating a control-packet exchange. CR user transmits data to other CR users using the maximum allowable power vector $\left(\mathbf{P}_{C, \beta}\right)$, derived in Section IV.

\section{B. Transmission Regions for a CR Node}

Each $\mathrm{CR}$ node $A$ is associated with a data region and a control region. Within these regions, other $\mathrm{CR}$ and $\mathrm{PR}$ users may exist. The data region of $A$ is defined as the area in which $A$ 's data transmission can be correctly received by another CR user. Let $r_{\text {data }}(A)$ be the radius of this region (how $A$ computes $r_{\text {data }}$ will be explained shortly). With probability $1-\beta$, the protocol protects all $\mathrm{PR}$ receptions that are within distance $r_{\text {data }}(A)$ and that share channels with $A$. The control region of $A$ is defined as the region in which $A$ 's control packets (e.g., RTS/CTS) can be correctly decoded. Let the radius of this region be $r_{c t r l}(A)$.

Let $a_{i}$ denote the maximum distance at which a CR user can correctly receive $A$ 's data transmission over channel $i$. The SINR requirement at a receiving $\mathrm{CR}$ node is given by:

$$
\mu_{i}^{*}=\frac{C\left(f_{i}\right) a_{i}^{-n} P_{C, \beta}^{(i)}}{\left(P_{t h}^{(i)}+P_{P R-C R}^{(i)}\right)}, \quad i=1, \ldots, M
$$

where $\mu_{i}^{*}$ is the SINR threshold required at the CR receiver to achieve a target bit error rate over channel $i, P_{t h}^{(i)}$ is the measured thermal noise over channel $i$, and $C\left(f_{i}\right) \stackrel{\text { def }}{=} \frac{G_{t}^{(i)} G_{r}^{(i)} l_{i}^{2}}{(4 \pi)^{2}\left(d_{o}^{(i)}\right)^{2-n}}$ is a frequency-dependent constant.

Rearranging (17),

$$
a_{i}=\sqrt[n]{\frac{C\left(f_{i}\right) P_{C, \beta}^{(i)}}{\mu_{i}^{*}\left(P_{t h}^{(i)}+P_{P R-C R}^{(i)}\right)}}, \quad i=1, \ldots, M .
$$

In (18), the CR-to-CR interference is ignored because of the aforementioned exclusive channel occupancy policy among CR users.

CR user $A$ maintains a list of available channels, denoted by $\operatorname{LAC}(A)$, which consists of the channels that are not currently used by any of $A$ 's CR neighbors (updated based on the overheard control messages). LAC $(A)$ conveys $A$ 's knowledge about the current spectrum usage in its vicinity. We set $A$ 's data transmission range to:

$$
r_{\text {data }}(A)=\min _{j \in \operatorname{LAC}(A)} a_{j}
$$

We impose the following constraint on $r_{c t r l}(A)$ to control the CR-to-PR interference:

Proposition 1: If $r_{c t r l}(A) \geq 2 \max _{j \in \operatorname{LAC}(A)} a_{j}$, then there is no overlap between the data region of $A$ and the data region of any other CR transmitter that overlaps with $A$ in one or more data channels.

Proof. By definition, for any channel $j \in \operatorname{LAC}(A), r_{\text {data }}(A) \leq$ $a_{j}$ and $r_{c t r l}(A) \geq 2 a_{j}$. Because of the exclusive channel occupancy, within the range $r_{c t r l}(A)$, no CR transmission other than $A$ 's can take place over channel $j$. Thus, the distance between $A$ and any other CR transmitter, say $C$, is at least $2 a_{j}$. If $C$ is outside the control region of $A$ and wants to reuse channel $j$, it will choose its $r_{\text {data }}(C)$ to be at most $a_{j}$. According to the proposition, $C$ will choose its $r_{c t r l}(C)$ to be at least $2 a_{j}$. Consequently, the data regions of $A$ and $C$ will not overlap, and only $A$ 's transmission will cause interference to PR users located in $A$ 's data region.

In Section VI, we study the impact of different settings of $r_{c t r l}(A)$ (as a function of $r_{\text {data }}(A)$ ) on the protocol's performance.

In general, the transmission range is a decreasing function of the transmission rate. Noting that the control channel requires a relatively low data rate, and consequently a low SINR threshold, the control range in Proposition 1 can be easily enforced through power control. Let $P_{c t r l}(A)$ be the power level that is needed to support the range $r_{c t r l}(A)$ over the control channel. In computing $P_{c t r l}(A)$, we account for the channel-specific $\mathrm{RF}$ attenuation and interference behavior. Formally, we set $P_{c t r l}(A)=\mu_{c}^{*} I^{(c)} /\left(C\left(f_{c}\right) r_{c t r l}(A)\right)$, where $f_{c}$ is the carrier frequency of the control channel and $I^{(c)}$ is the average noiseplus-interference over the control channel. Note that because neighboring CR users typically experience similar average PRto-CR interference [2], [7], it does not matter whether $I^{(c)}$ is computed at the transmitter or receiver since they are both within the same vicinity and would typically have similar view of the surrounding RF environment.

\section{Spectrum Access Mechanism}

We propose a spectrum access mechanism that enables the $\mathrm{CR}$ transmitter and receiver to agree on the set of channels to use. This mechanism also ensures that with probability $1-\beta$ the ensuing data transmission will not disturb any of the PR users in the vicinities of the CR transmitter and receiver. 
Suppose that CR user $A$ has data to transmit to CR user $B$. Let $R_{t o t}$ be the total rate demand (how to assign channels to support $R_{t o t}$ is described in Section V-D). If user $A$ does not sense a carrier over the control channel for a randomly selected backoff period, it proceeds as follows:

- If $\operatorname{LAC}(A)$ is empty or $B$ is busy (based on the overheard control messages), $A$ backs off and attempts to access the control channel later. Otherwise, $A$ computes its $r_{\text {data }}(A)$ according to (18) and (19). It also updates its $P_{c t r l}(A)$ according to Proposition 1, and then sends a Request-to-Send (RTS) message at power $P_{c t r l}(A)$ (the minimum power required to cover the control region). The RTS packet includes $\operatorname{LAC}(A)$, $P_{c t r l}$, the size of the upcoming data packet $(S)$, and $R_{t o t}$.

- The neighbors of $A$, other than $B$, that can correctly decode the RTS will stay silent until either they receive another control packet from $A$, denoted by DCTS (explained below), or until the time for the expected DCTS packet expires.

- Upon receiving the RTS packet, $B$ proceeds with the channel assignment process (described in Section V-D), whose purpose is to determine whether or not there exists a feasible set of channels $(\Omega(A, B))$ that can support the demand $R_{t o t}$.

- Depending on the outcome of the channel assignment process, $B$ decides whether or not $A$ can transmit. If not, then $B$ does not respond to $A$, prompting $A$ to back off, with an increased backoff duration as in typical CSMA/CA protocols, and retransmit later. Otherwise, $B$ sends a Clear-toSend (CTS) message to $A$, which contains $\Omega(A, B)$ and the duration $\left(T_{p k t}(A)\right)$ needed to reserve the assigned channels for the ensuing data transmission and associated ACK packet. The CTS implicitly instructs the CR neighbors of $B$ to refrain from transmitting over the set of assigned channels for the duration $T_{p k t}(A)$.

- Once $A$ receives the CTS, it replies back with a "DecidedChannels-to-Send" (DCTS) message, informing $A$ 's neighbors (who may not be able to hear the CTS) of $\Omega(A, B)$ and $T_{p k t}(A)$. Such a three-way handshake is typically needed in multi-channel CSMA/CA protocols (e.g., [5]-[7], [14], [16]).

- After completing the RTS/CTS/DCTS exchange, the transmission $A \rightarrow B$ proceeds. Once completed, $B$ sends back an ACK packet to $A$ over the channel in $\Omega(A, B)$ that has the highest rate. Figure 2 illustrates a time diagram for the controlpacket exchange.

Because there is no interference between data and control packets, a CR user that hears the RTS (CTS) packet defers its transmission only until the end of the handshake. This allows for more parallel transmissions to take place in the same vicinity.

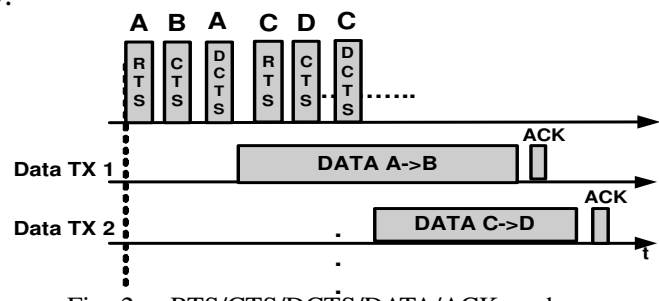

Fig. 2. RTS/CTS/DCTS/DATA/ACK exchange.

\section{Channel Assignment}

It is known that using more parallel channels for a transmission reduces the CR-to-PR interference per channel [3]. However, this may lead to channel over-assignment, which reduces the opportunity for assigning available channels to other CR transmitters (i.e., reduces the $\mathrm{CRN}$ spatial reuse). In our work, we statistically bound the CR-to-PR interference while using the minimum possible number of channels. Channel assignment (selection of $\Omega(A, B)$ ) depends on: (1) the maximum permissible power $\left(\mathbf{P}_{C, \beta}\right)$; (2) the list of common channels between $A$ and $B$, given by $\operatorname{LAC}(A, B) \stackrel{\text { def }}{=} \operatorname{LAC}(A) \bigcap \operatorname{LAC}(\mathrm{B})$; (3) the instantaneous interference level at $B$ over each channel in LAC $(A, B)$; (4) the channel gain between $A$ and $B$ (computed using the received signal strength of $A$ 's control packets); and (5) $R_{t o t}$. This $R_{t o t}$ is supported through the aggregate rate of all selected channels. Note that $\Omega(A, B) \subseteq \operatorname{LAC}(A, B)$.

Based on the above parameters, receiver $B$ acts as follows:

- When $B$ receives $A$ 's RTS, it first checks $\operatorname{LAC}(A, B)$ and removes any channel $i$ whose received SINR is less than $\mu_{i}^{*}$.

- $B$ sorts the rest of the available channels in a descending order of their data rates, calculated according to the receiver SINR and any predefined rate-vs-SINR relationships (e.g., Shannon's equation, staircase function, etc.). It then iteratively picks channels from the top of the sorted list until either the aggregate rate is satisfied, the sorted list is exhausted (i.e., no feasible channel assignment can be found), or the number of selected channels exceeds $n_{r}$. In the latter two cases, $B$ will not respond to $A$ 's RTS, prompting $A$ to back off and retransmit later. It is easy to show that this channel assignment is optimal in terms of minimizing the number of selected channels.

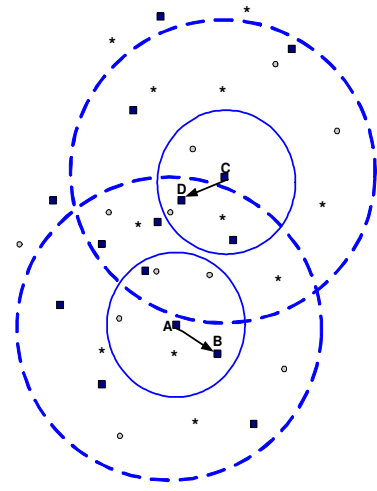

(a) Possible channel reuse

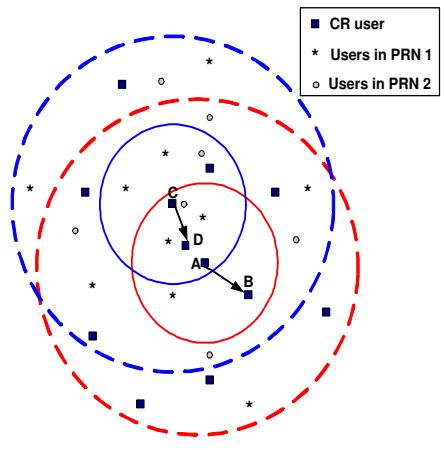

(b) Impossible channel reuse
Fig. 3. Scenarios in which a CR transmitter $C$ can/cannot reuse the channels assigned to $A$. Solid circles indicate data-transmission ranges, while dashed circles indicate control-transmission ranges.

Figure 3 depicts two scenarios for the operation of our MAC protocol. In the first scenario (Figure 3(a)), the two transmitters $A$ and $C$ cannot hear each other's control packets. So, according to Proposition 1, the transmissions $A \rightarrow B$ and $C \rightarrow D$ can overlap in their data channels. In Figure 3(b), node $C$ falls in the control region of node $A$ (and vice versa). The exclusive channel occupancy policy prevents $A$ and $C$ from using common channels. However, the two transmissions can proceed simultaneously if $A$ and $C$ can find non-intersecting channels to support their rates.

\section{Vi. Performance Evaluation}

We now evaluate the performance of our MAC protocol and study its effect on the performance of PR users. Our simulation programs are written in CSIM (a C-based process-oriented discrete-event simulation package).

In our analysis, we assumed that the interference at a receiver is equal to the sum of the interference powers of all other interferers within an interference radius $r_{c}$. Our simulations relax this assumption and account for all sources of interference, including those that are very far away from the receiver. We focus on one-hop CR communications and investigate the effect of coexistence between the CRN and the PRNs on network performance. Our performance metrics include the 




(a) $p_{\text {out }}$ vs. time $(\lambda=0.06)$

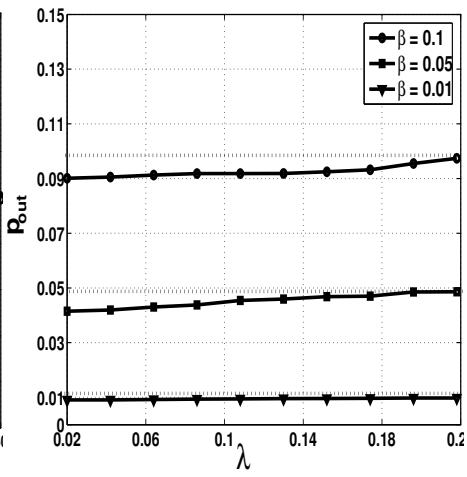

(b) pout vs. $\lambda$

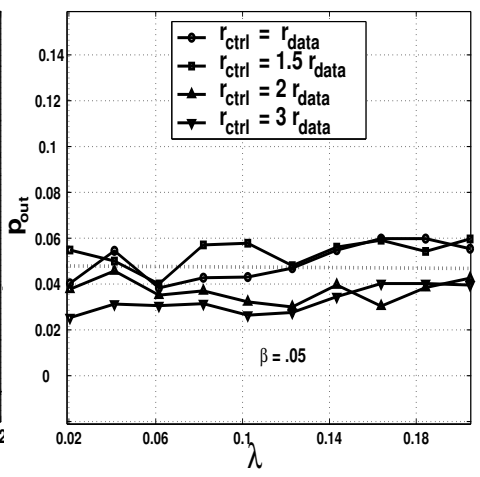

(c) $p_{\text {out }}$ (for different $r_{c t r l}($.$) ) vs. \lambda$

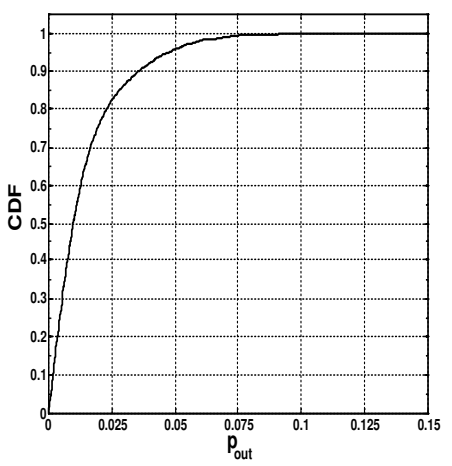

(d) CDF of observed $p_{\text {out }}(\beta=0.05)$

Fig. 4. Performance of a PRN.

outage probability for PR users, and the CRN goodput, defined as the average number of successfully received data packets per time slot. For simplicity, we consider a fixed-packet size ( 2 Kbytes) and a fixed rate demand $\left(R_{t o t}=10 \mathrm{Mbps}\right)$ for all CR users. A time slot corresponds to the transmission of one packet at $R_{\text {tot }}$.

\section{A. Simulation Setup}

We simulate a system consisting of 8 PRNs and 1 CRN. Users in each network are randomly distributed over a $500 \times 500$ meters $^{2}$ area. We study both uniform and non-uniform node deployments. Recall that our analysis assumed Poisson distributed PR users. The first 4 PRNs operate in the $900 \mathrm{MHz}$ band, occupying 4 non-overlapping $2.5 \mathrm{MHz}$ channels with $P_{L}^{(i)}=2 \times 10^{-9} \mathrm{~W}$. The other 4 PRNs operate in the 2.4 $\mathrm{GHz}$ frequency band, occupying 4 non-overlapping $2.5-\mathrm{MHz}$ channels with $P_{L}^{(i)}=1 \times 10^{-10} \mathrm{~W}$. The activity factors for the 8 PRNs are $0.1,0.2,0.3,0.4,0.1,0.2,0.3$, and 0.4 , respectively. The number of PR users in each PRN is 200 . The transmission power for each PR transmitter is $1 \mathrm{~W}$ and the antenna length $(D)$ is $5 \mathrm{~cm}$. We set the minimum distance between a PR receiver and the nearest PR interferer $\left(b_{i}\right)$ to 25 meters for all $i$. The CRN consists of 200 users. Each CR user generates packets according to a Poisson process with the same rate $\lambda$ for all users ${ }^{5}$. We set $n_{r}=4$. The signal propagation model in our simulations follows (1) with $n=4$. We set $\mu_{i}^{*}$ to $5 \mathrm{~dB}$ for all $i$. For all experiments, we select the value of $r^{*}$ (defined in Section IV) such that $F_{R_{\text {min }}}\left(r^{*}\right)=1-p^{*}=10^{-3}$. The reported results are averaged over 100 runs.

\section{B. Simulation Results}

We first investigate the effect of CR transmissions on the performance of PR users assuming uniform node deployment. Figure 4(a) illustrates $p_{\text {out }}$ versus time ${ }^{6}$ for two PRNs (1 and 6) with $\beta=0.05$. The reported results are cumulative over time, i.e., $0-100,0-200,0-300$, etc. It can be observed that $p_{\text {out }}$ is always less than $\beta=0.05$ for both PRNs. As time progresses, $p_{\text {out }}$ converges to a value less than 0.05 . These results are in line with the analysis in Section IV.

For the next experiments, we focus on the performance for PRN 1 (other PRNs depicted similar behaviors). Figure 4(b) demonstrates $p_{\text {out }}$ as a function of $\lambda$ at $\beta=0.01,0.05$,

\footnotetext{
${ }^{5}$ The stochasticity of the CRN traffic model plays a secondary role in our design. Hence, we opted for a simple model.

${ }^{6}$ All figures reporting $p_{\text {out }}$ show only the PR user that experienced the highest interference among all PR users in the given PRN.
}

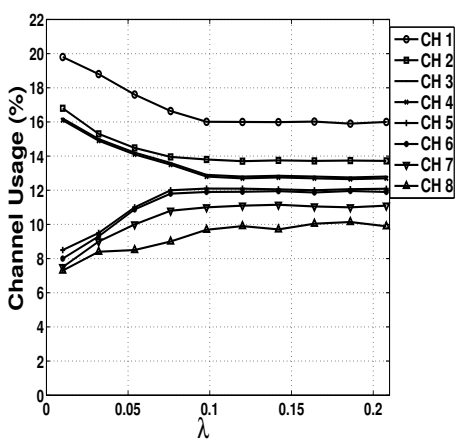

(a) CR channel usage vs. $\lambda$

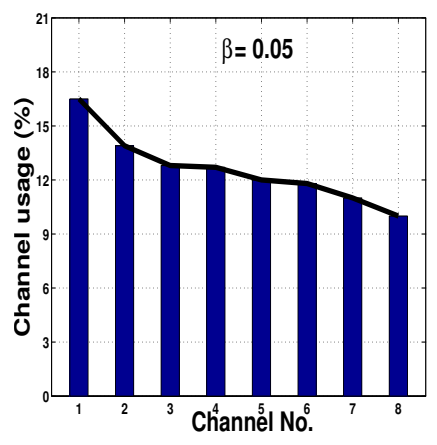

(b) CR channel usage (high load)
Fig. 5. Channel usage for the CRN.

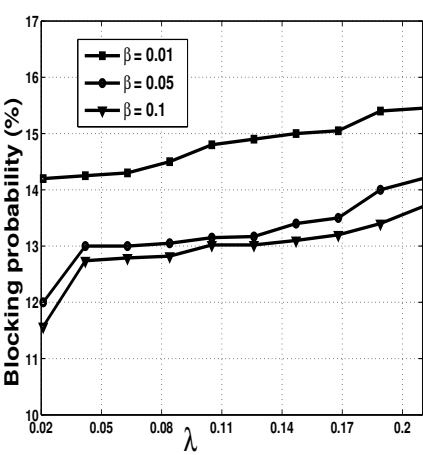

(a) CR blocking rate

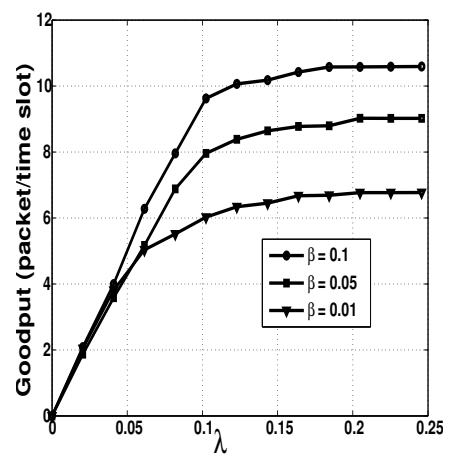

(b) CRN goodput for different values of $\beta$

Fig. 6. Performance of the CRN. and 0.1 . The results show that the bound on $p_{\text {out }}$ is always satisfied. The impact of different values of $r_{\text {ctrl }}($.$) on p_{\text {out }}$ is shown in Figure 4(c) with $\beta=0.05$. The figure illustrates that for $r_{c t r l}(.) \geq 2 r_{\text {data }}($.$) , the statistical guarantee is satisfied$ (i.e., $\left.p_{\text {out }}<0.05\right)$. However, for $r_{c t r l}()<.2 r_{\text {data }}($.$) , the$ statistical guarantee is not always satisfied. Experiments on different values of $r_{c t r l}($.$) indicate that a larger r_{c t r l}($.$) reduces$ the CRN goodput due to the decrease in the CRN spatial reuse (see [15] for details). Figure 4(d) shows the CDF of the observed $p_{\text {out }}\left(F_{p_{\text {out }}}\right)$ with $\beta=0.05$. The figure reveals that $<5 \%$ of the time the total interference power at a PR receiver exceeds the maximum tolerable interference (i.e., $\left.F_{p_{\text {out }}}(\beta=0.05)=\operatorname{Pr}\left[p_{\text {out }}<\beta\right]<0.95.\right)$. Thus, the statistical guarantee is satisfied.

For a given $\beta$, Figures 5(a) and (b) depict the channel usage, 


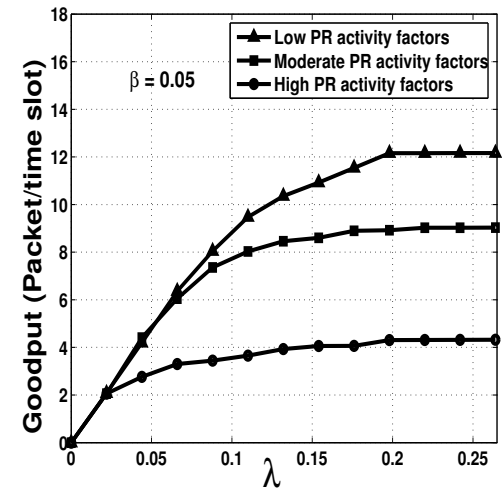

(a) CRN goodput for different $\alpha_{i}$

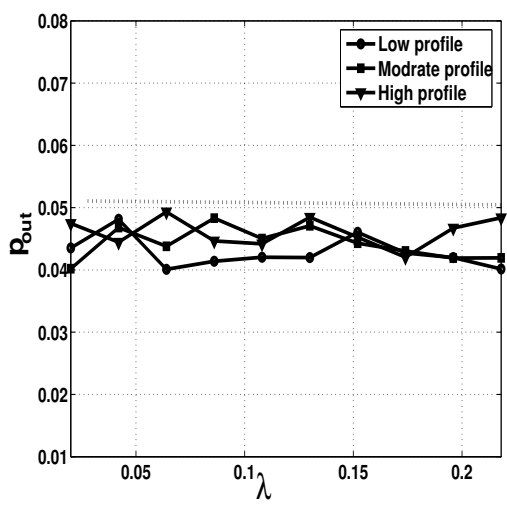

(b) $p_{\text {out }}$ vs. $\lambda$ (different $\alpha_{i}$ )

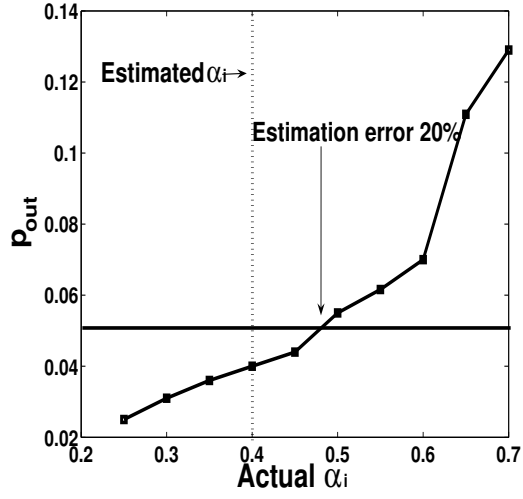

(c) $p_{\text {out }}$ under inaccurate $\alpha_{i}$

Fig. 8. Impact of activity profile on performance.

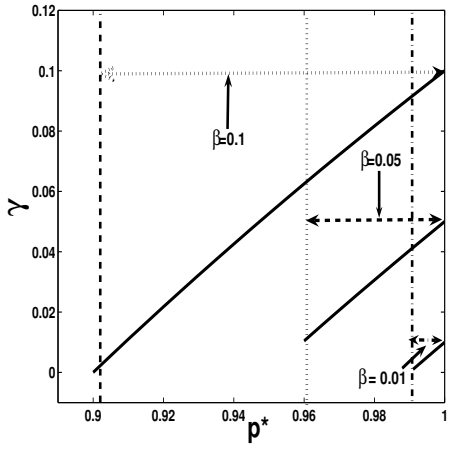

(a) $\gamma$ vs. $p^{*}$

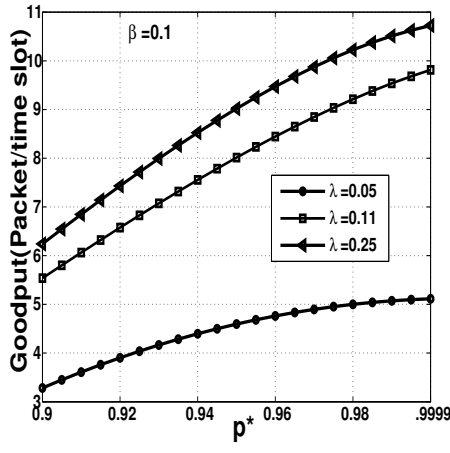

(b) Goodput vs. $p^{*}$
Fig. 7. Impact of selecting $p^{*}$.

defined as the fraction of time in which a specific channel is used for CR transmissions. These figures reveal that the carrier frequency and PRN activity factor affect channel usage (recall that the 8 PRNs differ in their $\alpha_{i}$ values). The smaller the value of $\alpha_{i}$, the higher is the channel utilization by the CRN. The CRN utilization of the lower four channels is higher than that for the higher four channels. Even though channels with lower carrier frequencies and smaller activity factors are favored for CR transmissions (lower attenuation), under moderate and high traffic loads, there are no significant differences in channel usage among all channels. Furthermore, channel usage remains fairly fixed in that traffic regime.

Figure 6(a) shows the effect of $\beta$ on the CRN connection blocking rate, defined as the fraction of $\mathrm{CR}$ transmission attempts that need to back off due to channel unavailability. As demonstrated, the blocking probability is smaller at larger $\beta$. This is because a larger $\beta$ increases the maximum allowable powers for CR users, and consequently decreases the required number of channels to support the aggregate rate demand. Figure 6(b) indicates that a larger value of $\beta$ results in improved CRN goodput. This can be deduced from (15) and (16), as larger $\beta$ results in increasing $\mathbf{P}_{C, \beta}$. Consequently, each CR user can use fewer number of channels and more CR transmissions can take place concurrently.

We also investigate the effect of selecting $p^{*}$ (equivalently $r^{*}$ ) (defined in Section IV) on the performance under different CR traffic loads. Figure 7(a) plots $\gamma$ over the feasible range of $p^{*}$ for different values of $\beta$. For $\beta=0.1$, Figures 7(a) and (b) indicate that using the largest possible value of $p^{*}$ (consequently the largest possible $\gamma$ ) results in improved CRN goodput. This can be deduced from (15) and (16), as larger $\gamma$ results in increasing
$\mathbf{P}_{C, \beta}$ for CR users. The increase in $\mathbf{P}_{C, \beta}$ reduces the number of channels assigned to a CR transmitter, which allows for more concurrent transmissions. Other results (shown in [15]) indicate that for all values of $p^{*}$ in Figure 7(a), the required guarantee is always achieved. Similar observations can be made for other values of $\beta$.

Next, we study the impact of $\alpha_{i}$ on performance. Three different activity profiles are simulated: high $\left(\alpha_{i}=0.8\right)$, moderate $\left(\alpha_{i}=0.4\right)$, and low $\left(\alpha_{i}=0.1\right)$. Figure 8(a) shows that the CRN goodput decreases for higher PRN activity profiles. Figure 8(b) indicates that the bound on $p_{\text {out }}$ is always achieved under different activity profiles. In Figure 8(c), we study the effect of inaccurately estimating $\alpha_{i}$. We operate our MAC protocol assuming an estimated activity factor of 0.4 , and vary its actual value in the simulations. It is noted that the required bound on $p_{\text {out }}$ is not satisfied when the actual $\alpha_{i}$ exceeds 0.4 by more than $20 \%$. Results indicate that $\alpha_{i}$ has to be conservatively estimated.

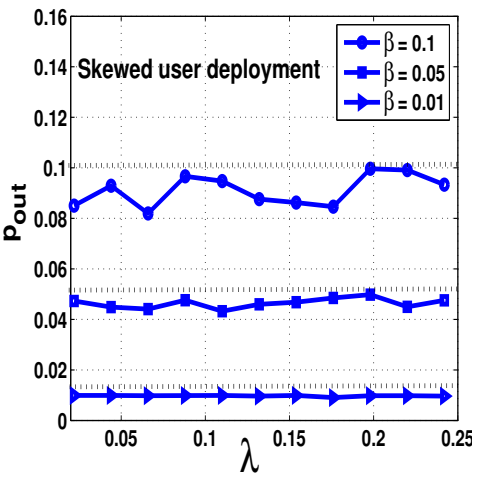

(a) $p_{\text {out }}$ vs. $\lambda$

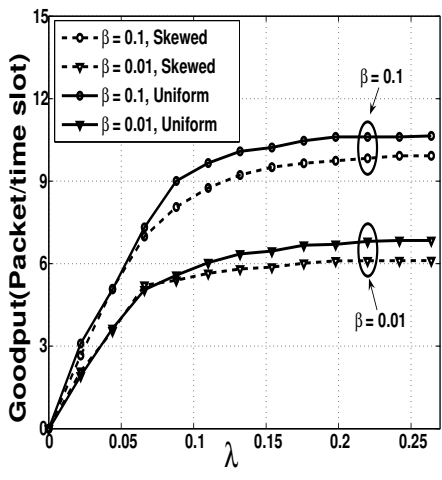

(b) CRN goodput for different $\beta$
Fig. 9. Performance under skewed and uniform user deployments.

Finally, we investigate the robustness of our protocol under a skewed user deployment. We assume that the network field is divided into four quadrants with respective user densities $60 \%$, $25 \%, 10 \%$, and $5 \%$. Figure 9(a) illustrates that the required bound on $p_{\text {out }}$ is still satisfied. However, Figure 9(b) shows that a minor reduction in the CRN goodput may occur compared with a uniform node deployment.

\section{CONCLUSION}

We investigated a statistical approach for dynamic spectrum access and radio resource management in opportunistic CRNs 
without assuming a predefined power mask. We first developed stochastic models for the PR-to-PR and the PR-to-CR interference under a Rayleigh fading channel model, and derived closed-form expressions for the mean and variance of each interference component. Furthermore, closed-form expressions were obtained for the characteristic function of the total interference under typical path loss exponents. Based on the derived interference models, we derived a closed-form expression for the maximum allowable powers for $\mathrm{CR}$ transmissions that ensure a statistical bound $\beta$ on $p_{\text {out }}$ for PR users. Then, we integrated our theoretical analysis in the design of a MAC protocol for opportunistic CRNs. Our protocol improves spectrum utilization while limiting the interference imposed on licensed users. Our simulation results show that our protocol statistically guarantees the performance of PR users under different CR traffic loads and for different values of $\beta$. Results also show that channel usage is reasonably balanced across various channels, even when the PR activity factors over such channels and the associated carrier frequencies are significantly different. Although uniform node deployment was used in our analysis, our simulations verified that the performance is not significantly impacted by the distributions of users in PRN/CRN.

\section{REFERENCES}

[1] "FCC, spectrum policy task force report, ET docket no. 02-155," Nov. 2002.

[2] I. Akyildiz, W.-Y. Lee, M.C. Vuran, and S. Mohanty, "Next generation dynamic spectrum access cognitive radio wireless networks: A survey," Computer Networks, vol. 50, no. 13, pp. 2127-2159, 2006.

[3] S. Haykin, "Cognitive radio: Brain-empowered wireless communications," IEEE Journal on Selected Areas in Communications, vol. 23, no. 2, pp. 201-220, Feb. 2005.

[4] S. Hijazi, B. Natarajan, M. Michelini, Z. Wu, and C. Nassar, "Flexible spectrum use and better coexistence at the physical layer of future wireless systems via a multicarrier platform," IEEE Wireless Communications, vol. 11, no. 2, pp. 8-14, April 2004.

[5] H. Bany Salameh, M. Krunz, and O. Younis, "Distance- and traffic-aware channel assignment in cognitive radio networks," in Proceedings of IEEE SECON08, June 2008.

[6] S. Sankaranarayanan, P. Papadimitratos, A. Mishra, and S. Hershey, "A bandwidth sharing approach to improve licensed spectrum utilization," in Proceedings of the IEEE DySPAN Conf., Nov. 2005, pp. 279-288.

[7] J. Zhao, H. Zheng, and G.-H. Yang, "Distributed coordination in dynamic spectrum allocation networks," in Proceedings of the IEEE DySPAN Conf., Nov. 2005, pp. 259-268.

[8] A. Sabharwal, A. Khoshnevis, and E. Knightly, "Opportunistic spectral usage: Bounds and a multi-band CSMA/CA protocol," IEEE/ACM Transactions on Networking, vol. 15, no. 3, pp. 533-545, 2007.

[9] V. Brik, E. Rozner, S. Banarjee, and P. Bahl, "DSAP: A protocol for coordinated spectrum access," in Proceedings of the IEEE DySPAN Conf., Nov. 2005, pp. 611-614.

[10] D. Cabric, S. Mishra, D. Willkomm, R. Brodersen, and A. Wolisz, "A cognitive radio approach for usage of virtual unlicensed spectrum," in Proceedings of the 14th IST Mobile and Wireless Communications Summit, June 2005

[11] L. Ma, X. Han, and C.-C. Shen, "Dynamic open spectrum sharing MAC protocol for wireless ad hoc networks," in Proceedings of the IEEE DySPAN Conf., Nov. 2005, pp. 203-213.

[12] T. C. Clancy, "Achievable capacity under the interference temperature model," in Proceedings of the IEEE INFOCOM Conference, May 2007, pp. 794-802.

[13] R. Menon, R. Buehrer, and J. Reed, "Outage probability based comparison of underlay and overlay spectrum sharing techniques," in Proceedings of the IEEE DySPAN Conf., Nov. 2005, pp. 101-109.

[14] T. Shu, S. Cui, and M. Krunz, "Medium access control for multi-channel parallel transmission in cognitive radio networks," in Proceedings of the IEEE GLOBECOM Conference, Nov. 2006.

[15] H. Bany Salameh, M. Krunz, and O. Younis, "Dynamic spectrum access protocol without power mask constraints," Tech. Rep. TR-UA-ECE-2008-1, University of Arizona, Aug. 2008, http://www.ece.arizona.edu/ krunz/Publications.htm/.

[16] Y. Yuan, P. Bahl, R. Chandra, T. Moscibroda, and Y. Wu, "Allocating dynamic time-spectrum blocks in cognitive radio networks," in Proc. of the ACM International Symposium on Mobile and Ad-Hoc Networking and Computing (MobiHoc), Sept. 2007.

[17] E. Sousa and J. Silvester, "Optimum transmission ranges in a directsequence spread-spectrum multihop packet radio network," IEEE Journal on Selected Areas in Communications, vol. 8, no. 5, pp. 762-771, June 1990.
[18] C. Hsu, A. Weit, D. Kuo, and C.-C.J., "A cognitive MAC protocol using statistical channel allocation for wireless ad-hoc networks," in Wireless Communications and Networking Conference (WCNC), March 2007, pp. $105-110$.

[19] Y. Xing, R. Chandramouli, S. Mangold, and S. Shankar, "Dynamic spectrum access in open spectrum wireless networks," IEEE Journal on Selected Areas in Communications, vol. 24, no. 3, pp. 626- 637, 2006.

[20] D. Cabric and R. Brodersen, "Physical layer design issues unique to cognitive radio systems," in Proceedings of the IEEE International Symposium on Personal, Indoor and Mobile Radio Communications (PIMRC), Sept. 2005

[21] Application Note (AN-SR-7), "Building a versatile low latency cognitive radio for multi-mission applications with the ICS-572," Tech. Rep., Sep 2003, http://www.gefanucembedded.com/news-events/whitepapers/3067.

[22] C. Yu, K. Shin, and L. Song, "Link-layer salvaging for making routing progress in mobile ad hoc networks," in Proc. of the ACM International Symposium on Mobile and Ad-Hoc Networking and Computing (MobiHoc), May 2005, pp. 242-254.

[23] T. Rappaport, Wireless Communications-Principles and Practice, Prentice-Hall Press, 2001, 2nd edition.

[24] S. Selby, Basics Mathematical Tables, The Chemical Rubber Co., 17th edition.

[25] C. Stevenson, C. Cordeiro, E. Sofer, and G. Chouinard, "Functional requirements for the 802.22 WRAN standard r47," 2005.

[26] P. Pawelczak, R. Prasad, X. Xai, and I. Niemegeers, "Cognitive radio emergency networks - requirements and design," in Proceedings of the IEEE DySPAN Conf., Nov. 2005, pp. 601-606.

\section{APPENDEX \\ PROOF OF THEOREM 1}

Let $P_{r, j \mid y}^{(i)}$ denote the received power $P_{r, j}^{(i)}$ conditioned on $\xi^{(i)}=y$. Because the probability that the distance between a PR user and a CR user is less than $d_{o}{ }^{(i)}$ is approximately zero, the characteristic function $(\mathrm{CF})$ of $P_{r, j \mid y}^{(i)}\left(\phi_{P_{r, j \mid y}^{(i)}}(\omega) \stackrel{\text { def }}{=}\right.$ $\left.E\left[e^{j \omega P_{r, j}^{(i)}} \mid \xi^{(i)}=y\right]\right)$ can be written as:

$$
\phi_{P_{r, j \mid y}^{(i)}}(\omega) \approx \int_{d_{o}^{(i)}}^{r_{c}} e^{j \omega P_{o}^{(i)}\left(\frac{r}{d_{o}^{(i)}}\right)^{-n} y} f_{R}(r) d r .
$$

Substituting (3) into (20) and algebraically manipulating the result, we obtain:

$$
\phi_{P_{r, j \mid y}^{(i)}}(\omega)=2\left(\frac{d_{o}^{(i)}}{r_{c}}\right)^{2} \int_{1}^{\frac{r_{c}}{d_{o}^{(i)}}} x e^{j \omega P_{o}^{(i)} x^{-n} y} d x .
$$

The CF of $P_{r, j}^{(i)}, \phi_{P_{r, j}^{(i)}}(\omega)$, can be obtained by removing the conditioning in (21) and algebraically manipulating the result:

$$
\begin{aligned}
\phi_{P_{r, j}^{(i)}}(\omega) & =\int_{0}^{\infty} \phi_{P_{r, j \mid y}^{(i)}}(\omega) f_{\xi_{j}^{(i)}}(y) d y \\
& =2\left(\frac{d_{o}^{(i)}}{r_{c}}\right)^{2} I_{C R, i}(n, \omega) .
\end{aligned}
$$

Recall that the number of PR users in the plane is Poisson distributed with mean of $\rho_{i}$ users per unit area. Because each PR user behaves as an ON/OFF source with activity factor $\alpha_{i}$, the number of active PR transmitters in $R_{c}\left(N_{i}\right)$ forms a Poisson random variable with mean of $\alpha_{i} \rho_{i}$ active users per unit area. Conditioned on $N_{i}=n_{i}$, the $\mathrm{CF}$ of $P_{P R-C R}^{(i)}$ is given by:

$$
E\left[e^{j \omega P_{P R-C R}^{(i)} \mid N_{i}}=n_{i}\right] \approx\left(\phi_{P_{r, j}^{(i)}}(\omega)\right)^{n_{i}} .
$$

By removing the conditioning in (23), the CF of the total PRto-CR interference over channel $i, \phi_{P_{P R-C R}^{(i)}}(\omega)$, is given by:

$$
\phi_{P_{P R-C R}^{(i)}}(\omega)=\sum_{n_{i}=0}^{\infty} \frac{e^{-\alpha_{i} \rho_{i} R_{c}}\left(\alpha_{i} \rho_{i} R_{c}\right)^{n_{i}}}{n_{i} !}\left(\phi_{P_{r, j}^{(i)}}(\omega)\right)^{n_{i}}(24)
$$

By summing the series, (24) can be reduced into (4) in Theorem 1 . 